\title{
Study on the effect of steel ball abrasion on surface hardness of workpiece in strengthen grinding processing
}

\author{
Jinrui Xiao ${ }^{1,2, a}$, Xiaochu Liu ${ }^{1,2, b^{*}}$, Zhongwei Liang, 2, c, Wenbo Zhou ${ }^{1,2, d}$, Zhuan \\ Zhao $^{1,2, e}$ and Changhong Liu ${ }^{1,2, f}$ \\ ${ }^{1}$ School of Mechanical and Electric Engineering, Guangzhou University, Guangzhou 510006, China \\ ${ }^{2}$ The Guangzhou Key Laboratory for Strengthen Grinding and High Performance Machining of \\ Metal Material, Guangzhou University, Guangzhou 510006, China \\ axjr640@163.com, ’gdliuxiaochu@163.com, 'Izwstalin@126.com, d1158373689@qq.com, \\ e598557559@qq.com, ${ }^{\mathrm{f}} 69481006 @ q q . c o m$,
}

*Corresponding author: Xiaochu Liu, email: gdliuxiaochu@163.com

Keywords: strengthen grinding, steel ball abrasion, surface hardness, stress-strain.

Abstract. Aiming at the problem of the workpiece surface hardness influenced by the fatigue wear of steel ball during strengthen grinding processing, experimental and mechanical analysis were performed. The relationships between the radius of steel ball and the stress and strain in the machining process were analyzed, the mathematical model of the workpiece surface hardness and residual stress was established, and the results showed that the surface residual stress and the surface hardness increment decreased with the increase of steel ball abrasion.

\section{Introduction}

The strengthen grinding of metallic materials is a new technology of green energy saving, anti fatigue and life prolonging, which was first put forward by Professor Liu of Guangzhou University. It works by spraying the gas liquid solid three phase jet flow, which composed of strengthening abrasive and high pressure gas, to the metal workpiece surface. It can make plastic strengthen and micro-cutting grinding and tribo-chemistry modification come true at the same time. Moreover, the wear-resisting property, fatigue life and reliability of workpiece will be improved significantly [1]. However, affecting by a series of complex mechanical and chemical effects, such as three phase mixing flow field, abrasive particle impact, friction and micro-cutting, the cold hardening and brittleness of steel ball will be formed due to over strengthening, and fatigue spalling on the surface of steel ball will appear caused by the cyclic alternating stresses during strengthen grinding processing. Then, it will be invalidated, and affects the hardness of machined surface and reduces the fatigue resistance directly [2,3]. Therefore, it is of great significance to explore the effect of the steel ball abrasion on the surface hardness of the workpiece in the strengthen grinding processing.

\section{Specimen Preparation and Experimental Method}

The object of this paper is the $6207 \mathrm{~N}$ inner bearing ring after heat treatment. Its density is 7850 $\mathrm{kg} / \mathrm{m} 3$, Young's modulus is $217 \mathrm{GPa}$, tensile strength is $2352 \mathrm{MPa}$, and yield limit is $1744 \mathrm{MPa}$. The processing equipment is bearing strengthen grinding machine, and the parameters are set as follows: the abrasive jet pressure is $0.6 \mathrm{MPa}$, the injection angle is 45 degrees, the distance is $50 \mathrm{~mm}$, and the rotational speed is 100r/min, as shown in Fig. 1.

The strengthening abrasive is made up of nitrogen-rich liquid, brown corundum grinding mill and steel ball according to the mass ratio of $1: 2: 3$. Among them, the steel ball diameter is $0.2 \mathrm{~mm}$, the average hardness is $63 \sim 65 \mathrm{HRC}$, the density is $7890 \mathrm{~kg} / \mathrm{m}^{3}$, the carbon content is $0.1 \%$, the manganese content is $0.3 \%$, the silicon content is $0.35 \%$, and the chromium content is $1.5 \%$. 10 bearing inner ring were taken and numbered 1 10, and the surface hardness of the raceway was measured. One inner ring was processed after 25 times cycle of strengthening abrasive. The raceway surface hardness of samples after processing was measured. 


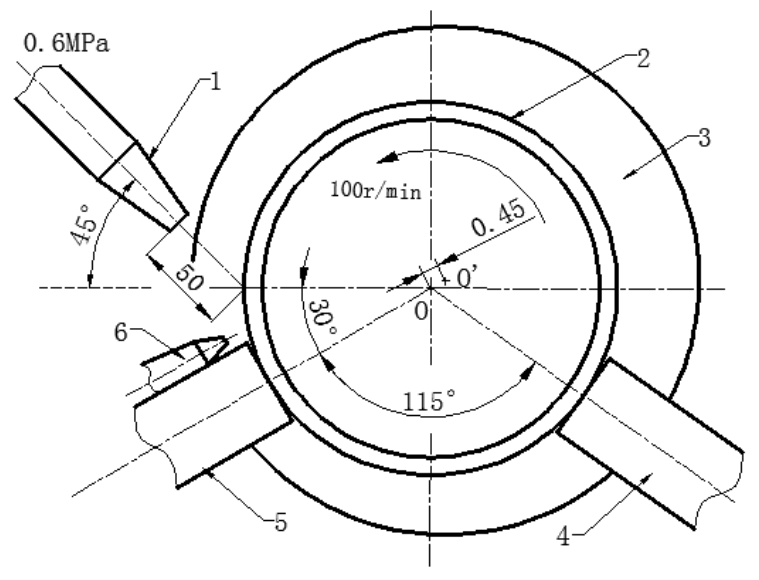

1- High-pressure nozzle ; 2-Bearing ring ; 3- Electromagnetic chuck ;

4- Right support ; 5-Left support ; 6- Nitrogen nozzle ;

Fig. 1 the processing technological conditions of reinforced grinding

\section{Results and discussion}

The average value of the bearing ring raceway surface hardness is $61.7 \sim 62.5 \mathrm{HRC}$ before processing, and up to $62.3 \sim 63.3 \mathrm{HRC}$ after processing, the maximum increment is of $1.3 \mathrm{HRC}$. The increment of the surface hardness of the workpiece is mainly determined by the stiffness of the steel ball and the original hardness difference between the steel ball and the workpiece under the same processing conditions. When the steel ball was worn, its stiffness and surface hardness decreased, and resulting in the decreasing of intensification effect on the workpiece surface, high hardness lattice formed was reduced, and the processed workpiece surface hardness increment decreased. It could be speculated that the steel ball was in a state of micro wear before 125 cycles, and was in a state of rapid wear after 125 cycles, then achieved a new balance after 200 cycles, as shown in Fig. 2.

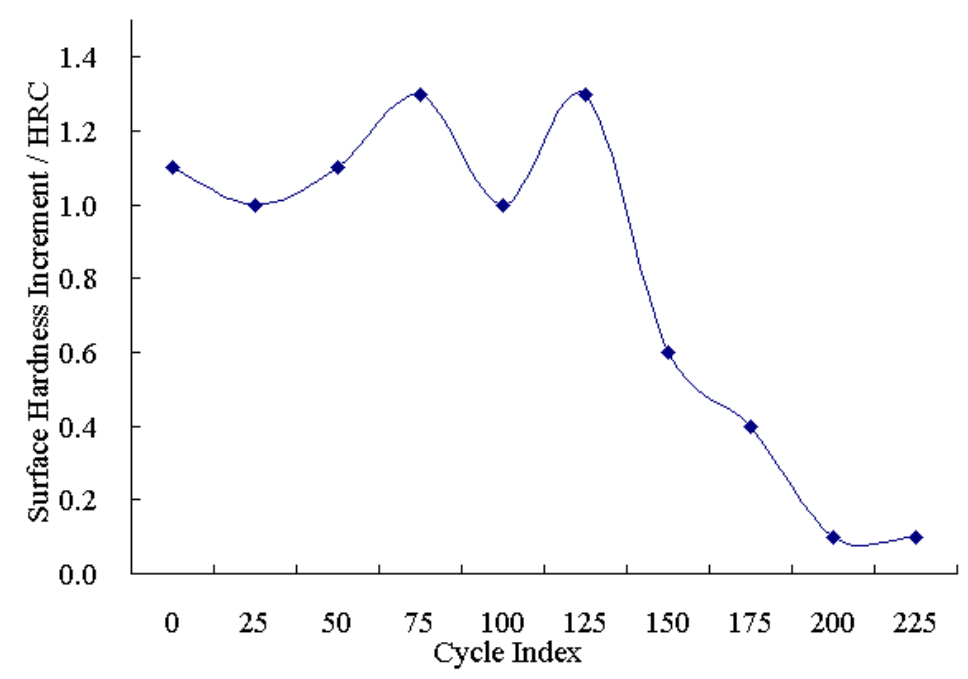

Fig. 2 Bearing ring surface hardness increment after processing

The impact occurred on the surface of steel ball and bearing raceway in the normal direction. Combining with Hertz contact theory and the principle of mechanical and chemical strengthening grinding[4], the impact force of abrasive on metal workpiece could be achieved, such as formula (1).

$$
F_{n}=1.2 R^{2} \rho^{0.6} \frac{1}{\left[\frac{1-v_{1}^{2}}{E_{1}}-\frac{1-v_{2}^{2}}{E_{2}}\right]^{0.4}}(v \sin \alpha)^{1.2}
$$


Here $\mathrm{R}$ denotes the average radius of steel ball, $\rho$ denotes the average density, $v_{1}$ and $v_{2}$ respectively denotes the Poisson's ratio of steel ball and inner bearing ring, E1 and E2 respectively denote the elastic modulus of the steel ball and bearing ring.

When completely plastic deformation occurred on the surface of bearing ring raceway during the impact processing, the reaction force on the steel ball $\left(F_{n}\right)$ was 3 times of the yield limit stress of the bearing raceway $\left(\sigma_{0}\right)$. Integrating the dynamic equilibrium motion equation, and formula (2) could be taken[5].

$$
\frac{\bar{Z}}{R}=\left(\frac{2}{3}\right)^{0.5}\left(\frac{\rho v^{2}}{F_{n}}\right)^{0.5}=\left(\frac{2}{3}\right)^{0.5}\left(\frac{\rho v^{2}}{3 \sigma_{0}}\right)^{0.5}
$$

Here $\bar{Z}$ denotes the depth of pits on the specimen surface

If the pits on the surface of bearing ring are regarded as circular hollow thick sphere that consisted of uniform force, then the strain along radial was equivalent. Along depth direction, the expressions of the stress distribution along the radial direction and the depth direction of the circular hollow thick sphere were shown as formula (3) (4) respectively when $\mathrm{a}<\mathrm{Z}<\mathrm{a}+\mathrm{h}[6]$.

$$
\begin{aligned}
& \sigma_{r}=-2 \sigma_{0} \ln \left(\frac{\mathrm{h}+a}{Z}\right)+\frac{1}{3} \\
& \sigma_{z}=-2 \sigma_{0} \ln \left(\frac{\mathrm{h}+a}{Z}\right)-\frac{2}{3}
\end{aligned}
$$

Here $\mathrm{h}$ denotes the plastic deformation depth of the machined bearing surface.

The residual stress distribution along the depth of the pit was obtained by the formula (3) and (4) and Hertz theory, as following:

$$
\begin{aligned}
& \left(\sigma_{r}\right)_{r}=\left[-2 \ln \left(\frac{h+a}{z}\right)+\frac{1}{3}\right] \sigma_{0}+\left\{(1+v)\left[1-\frac{z}{a} \tan ^{-1}\left(\frac{a}{z}\right)\right]-\frac{1}{2}\left(1+\frac{z^{2}}{a^{2}}\right)^{-1}\right\} \times 1.2 k_{1} R^{2} \rho^{0.6} \\
& \left(\sigma_{z}\right)_{z}=\left[-2 \ln \left(\frac{h+a}{z}\right)-\frac{2}{3}\right] \sigma_{0}+\left(1+\frac{z^{2}}{a^{2}}\right)^{-1} \times 1.2 k_{1} R^{2} \rho^{0.6}
\end{aligned}
$$

Under the same conditions, with the increase of cycle number, the alternating stress and reinforcing led to the abrasion phenomenon of fatigue and brittle fracture. Then, the average radius and density of steel ball will be decreased, and leading to the decreasing of the depth, the maximum radius and the depth of pits. Finally, the residual compressive stress on the surface of the workpiece was reduced.

It was assumed that the stress-strain relation of the material satisfied the power-law hardening law, and the residual compressive stress field satisfied the two-dimensional axial condition. Then, the expressions of residual compressive stress $\sigma$ and surface hardness $\mathrm{H}$ and $\mathrm{C}$ can be obtained [7], as following:

$$
\left(\frac{5}{17} \sigma_{1} H\right)^{n}=\sqrt[n]{\frac{\sigma}{\sigma_{1}(e-1)}}+0.08
$$

Here $\sigma_{1}$ denotes the strength coefficient of workpiece material and $n$ denotes the strain hardening exponent.

There is a positive correlation between the surface hardness and surface residual compressive stress according to the formula (7). It could be known that the plastic deformation, such as surface relaxation, surface peeling, particle crushing etc, appeared because of the reciprocating impact with the enhanced of recycling in the same conditions of mechanical and chemical strengthen grinding according to 
formula (5) and (6). Then, the average radius and density of the steel ball were reduced, and the residual compressive stress that formed during process was decreased. Then the $H$ decreased with the decreased of $\sigma$, while the surface hardness and surface residual compressive stress of workpiece were positively correlated. Therefore, with the increasing of cycle index, the steel ball would be worn. Finally, the surface residual compressive stress and hardness increment would be reduced.

\section{Conclusions}

Based on the test of the steel ball wear effects on workpiece surface hardness increment, the relationship between the radius, the stress and the strain of steel ball during the processing were analyzed, and the relationship between the surface hardness and residual stress was established. Finally, the conclusion was drawn that the abrasion of steel ball would reduce the surface residual compressive stress and hardness increment.

\section{Acknowledgements}

This work was financially supported by the National Natural Science Foundation of China (U1601204) and the Innovative Academic Team Project of Guangzhou Education System (1201610013).

\section{References}

[1] Chuanjian Liu, Xiaochu Liu, Wenxiong Li, et al. Numerical simulation of collision in strengthening and polishing of bearing ring raceway [J]. Bearing, 2010 (12): 21-24.

[2] Jinrui Xiao, Xiaochu Liu, Bihong Xie, et al. Study on the Effect upon Wear of Steel Ball from Injection Pressure in Reinforced Grinding Processing[C]. Advanced Materials Research. Trans Tech Publications, 2014, 915: 189-192.

[3] Xiaochu Liu, Jinrui Xiao, Jianwen Zhang, et al. Study on the Effect upon Wear of steel ball from Jetting Time in Reinforced Grinding Processing [J]. Manufacturing Technology \& Machine Tool, 2015 (3): 112-114.

[4] Tonglin Li, Yuanyu Yin. Elastic plastic mechanics[M]. Wuhan: China University of Geosciences Press, 2006:43-48.

[5] Al-Hassani STS. Mechanical aspects of residual stress development in shot peening[A ]. Proceedings of the 1st international conference on shot peening (ICSP1), Paris: Pergamon Press, $1981: 583-602$.

[6] Al-Hassani STS. An engineering approach to shot peening mechanics[A]. Proceedings of the 2th international conference on shot peening(ICSP2), Chicago, USA, 1984: 275-282.

[7] Lihua Lin, Ligong Chen, Mingyuan Gu. A new method for determining residual stress--A spherical indenter pressed on the structure surface and added a additional stress field[J]. Journal of mechanical strength, 1998, 20(4): 303-306. 